\title{
On the Origin of Exponential Disks at High Redshift
}

\author{
Bruce G. Elmegreen \\ IBM Research Division, T.J. Watson Research Center, P.O. Box 218, Yorktown Heights, \\ NY 10598,USA, bge@watson.ibm.com \\ Debra Meloy Elmegreen, David R. Vollbach, Ellen R. Foster, and Thomas E. Ferguson \\ Vassar College, Dept. of Physics \& Astronomy, Box 745, Poughkeepsie, NY 12604; \\ elmegreen@vassar.edu,davollbach@vassar.edu,elfoster@vassar.edu,thferguson@vassar.edu
}

\begin{abstract}
The major axis and ellipse-fit intensity profiles of spiral galaxies larger than $0.3^{\prime \prime}$ in the Hubble Space Telescope Ultra Deep Field (UDF) are generally exponential, whereas the major axis profiles in irregular disk galaxies, called clumpclusters in our previous studies, are clearly not. Here we show that the deprojected positions of star-forming clumps in both galaxy types are exponential, as are the deprojected luminosity profiles of the total emissions from these clumps. These exponentials are the same for both types when normalized to the outer isophotal radii. The results imply that clumps form or accrete in exponential radial distributions, and when they disperse they form smooth exponential disks. The exponential scale lengths for UDF spirals average $\sim 1.5 \mathrm{kpc}$ for a standard cosmology. This length is smaller than the average for local spirals by a factor of $\sim 2$. Selection effects that may account for this size difference among spirals are discussed. Regardless of these effects, the mere existence of small UDF galaxies with grand-design spiral arms differs significantly from the situation in local fields, where equally small disks are usually dwarf Irregulars that rarely have spiral arms. Spiral arms require a disk mass comparable to the halo mass in the visible region - something local spirals have but local dwarfs Irregulars do not. Our UDF result then implies that galaxy disks grow from the inside out, starting with a dense halo and dense disk that can form spiral arms, and then adding lower density halo and disk material over time. Bars that form early in such small, dense, gas-rich disks should disperse more quickly than bars that form later in fully developed disks.
\end{abstract}

Subject headings: galaxies: formation — galaxies: evolution — galaxies: highredshift 


\section{Introduction}

The origin of exponential disks has been difficult to observe directly because the earliest examples of clear spiral galaxies already have them (O’Neil, Bothun, \& Impey 2000; Pérez 2004; Elmegreen, Elmegreen, \& Sheets 2004; Toft et al. 2005). Exponential disks have also been difficult to model in cosmology simulations (van den Bosch 2001; Robertson et al. 2004), although models with high numerical resolution (Governato et al. 2004), star formation feedback (Westera et al. 2002; Abadi et al. 2003), or nuclear black-hole feedback (Robertson et al. 2005) have made exponential disks.

In two previous surveys, we distinguished between spiral galaxy disks, which have bulges, spiral arms, and approximately exponential profiles, and highly irregular disks, which we called clump cluster galaxies (Elmegreen, Elmegreen \& Sheets 2004; Elmegreen et al. 2005b). The association of clump clusters with disks was made on the basis of models for the distribution of axial ratios (Elmegreen, Elmegreen, \& Hirst 2004a; Elmegreen \& Elmegreen 2005; Elmegreen et al. 2005b). Edge-on clump clusters are apparently chain galaxies, which also have no central bulges or exponential disks, and have similar clumps (Elmegreen, Elmegreen, \& Hirst 2004a). In a study of 10 clump-cluster galaxies, the average clump mass was found to be $\sim 6 \times 10^{8} \mathrm{M}_{\odot}$ and the average clump diameter was $1.8 \mathrm{kpc}$ (Elmegreen \& Elmegreen 2005).

Clump clusters, by definition, do not have central light concentrations resembling bulges, nor do they have exponential disks along any radial profile. Some clump clusters, however, have nearly exponential disks in their average radial light profile, even though the major axis profile is irregular. Generally, the elliptical contours for clump cluster galaxies cannot be determined automatically by the IRAF routine ellipse because the galaxy disks are too irregular. Still, we can use a constant ellipticity and position angle to determine the average radial profile, and obtain the values of the center, ellipticity and position angle from the outer $2 \sigma$ contour, where $\sigma$ is the image noise level. Three examples of intensity profiles are shown in Figure 1, along with the galaxy images. On the left is a spiral with exponential profiles for both the major axis (top panel) and the ellipse fits. In the central column is a clump cluster with a typically irregular major axis profile but a somewhat exponential elliptically-averaged profile. On the right is another clump cluster with no exponential at all.

Exponential light profiles may be the result of cosmological collapse during galaxy formation (Freeman 1970; and see references above) or the result of radial flows in viscous, starforming disks (e.g., Lin \& Pringle 1987; Yoshii \& Sommer-Larsen 1989; Zhang \& Wyse 2000; Ferguson \& Clarke 2001). In the collapse simulations, the gas may be accreted smoothly from the halo (Murali et al. 2002; Westera et al. 2002; Sommer-Larsen, Götz, \& Portinari 
2003; Keres et al. 2005) or erratically from cannibalized dwarf companions (Walker, Mihos, \& Hernquist 1996; Abadi et al. 2003) and intergalactic clouds (Brook et al. 2004).

In the viscous models, the inner regions tend to move in, making a central concentration, while the outer regions tend to spread out, making an exponential in the outer disk. The time scale for the development of the outer exponential can be long, comparable to the star formation time, if the initial disk is significantly flatter than exponential or if it is highly irregular. The presence of exponential disks in local dwarf irregular galaxies (Hunter \& Elmegreen 2005), where there is little shear for viscous effects, and the early appearance of exponentials in high-redshift spirals, suggest that this profile is related to galaxy assembly, not shear.

In order to understand star formation in the disks of young galaxies, we have been studying the properties of the bright blue clumps which dominate the appearance of clump clusters (Elmegreen \& Elmegreen 2005). Clump masses can be as large as $10^{9} \mathrm{M}_{\odot}$. Most of these clumps have average densities only slightly larger than the critical tidal densities in their disks, so they should eventually disperse. Many also have tails or other structures which make them look like they are in the process of dispersing. As these galaxies also look very young (van den Bergh et al. 1996; Conselice et al. 2004), clump dispersal in clump-cluster galaxies could offer a clue to the origin of exponential disks.

Here we show that even though the radial light profiles of clump cluster galaxies are not exponential, the average radial profiles of the clump positions are exponential, as are the average radial profiles of the integrated clump fluxes. Thus, these galaxies may evolve into exponential disks by the dissolution of the observed clumps. In that case, it may be concluded that cosmological accretion promotes exponential disks whether the gas comes in smoothly or in the form of giant clumps. If the accretion is smooth, then the disk has to be highly turbulent so that gravitational instabilities form clouds and stellar clumps (Immeli et al. 2004) with a Jeans mass as large as a clump mass.

\section{Small Exponential Scale Lengths in UDF Spirals}

The spiral or disk-like galaxies in our morphological catalog of the Hubble Space Telescope Ultra Deep Field (UDF; Beckwith et al. 2005) were fit to elliptical isophotes using IRAF (Elmegreen et al. 2005b). We considered only objects larger than 10 pixels in diameter on the $i_{775}$ image. Those which had exponential light profiles, spiral arms, and central

bulges were designated as spirals (269 galaxies) and those which did not were designated as clump clusters (178 of them). All UDF spirals have an exponential disk with a central bulge, 
although most of these bulges are small.

For the spirals, we determined the exponential scale lengths in pixels using the median value of the local slope on a log-linear plot of ellipse-fit intensity versus radius in $i_{775}$ band. This wavelength was chosen because it has the deepest UDF exposures. The central pixel was not included in the ellipse fits. The median technique avoids the small scale length of the bulge region and possible scale length shifts in the far outer region. The result is a good approximation to the scale length of the main-disk exponential. Figure 2 shows 4 examples of the radial intensity profiles. The dotted lines are the fits and the dashed horizontal lines are the $2 \sigma$ background noise levels. We do not discuss here double exponential disks or disk truncations, such as those observed in local galaxies by Kregel \& van der Kruit (2004), Erwin, Beckman, \& Pohlen (2005), and others. Such profiles are present in our UDF sample and they have also been noted for other high-redshift galaxies by Pérez (2004).

Figure 3 shows the distribution function of the spiral galaxy scale lengths measured in pixels. Although the UDF spirals probably span a wide range of redshifts, this distribution function has physical meaning because the conversion between angular size and physical size is relatively flat at redshifts from 0.5 to 5 . The conversion factor is shown in Figure 4 for the WMAP $\Lambda$ CDM cosmology (Spergel et al. 2003). The peak at $\sim 7$ pixels in Figure 3 converts into 0.21 arc seconds for the ACS camera, in which case Figure 4 (dashed line) suggests that most spirals have an exponential scale length of around $1.5 \mathrm{kpc}$, to within a factor of $\sim 1.5$.

This resultant average of $\sim 1.5 \mathrm{kpc}$ for the UDF is smaller than the average exponential scale length in local spiral galaxies. van der Kruit (1987) obtained a local scale length distribution with a peak at $3 \pm 2 \mathrm{kpc}$ for 42 galaxies having known distances; the 31 spirals in his sample had an average scale length of $4 \mathrm{kpc}$ and the 11 dwarfs had an average scale length of $1 \mathrm{kpc}$. de Jong (1996a) observed B and K-band scale lengths somewhat uniformly distributed between 1 and $7 \mathrm{kpc}$ for 86 local galaxies, but the volume-corrected distribution, which included substantial corrections for missing small galaxies, peaked at $1.5 \mathrm{kpc}$ and extended from 0.8-11 kpc. Courteau (1996) found a scale length distribution for 290 local Sb-Sc galaxies that peaked at $\sim 3.5 \mathrm{kpc}$ with a range between 1.5 and $6 \mathrm{kpc}$. McGaugh \& Bothun (1994) obtained a similar range of exponential scale lengths (1.2-6.3 kpc) for low surface brightness galaxies, as did de Blok, van der Hulst, \& Bothun (1995), who got a median of $3.2 \mathrm{kpc}$. For local dwarf galaxies, Hunter \& Elmegreen (2005) obtained an average V-band scale length of $1 \mathrm{kpc}$ for 94 galaxies of type Im, and $1.7 \mathrm{kpc}$ for 18 galaxies of type Sm. Similar sizes were obtained in a survey of 171 dwarf galaxies by Swaters \& Balcells (2002), who found the distribution of scale lengths peaked at $\sim 1 \mathrm{kpc}$ and extended from approximately 0.2 to $4 \mathrm{kpc}$. Evidently, the UDF spirals are systematically smaller than local spirals by a factor of $\sim 2$, and comparable in size to local spiral dwarfs (type Sm). The local 
dwarf Irregulars (type Im) may be slightly smaller than the UDF spirals.

The exponential scale length is a better measure of spiral galaxy size than outer isophotal radius, which depends on the surface brightness relative to the survey limit and varies with redshift through cosmological dimming. The scale length may depend on redshift through band shifting, as local disks have 20\% larger B band scale lengths than K-band (de Jong 1996b; de Grijs 1998). Such color gradients typically come from star formation, metallicity, and extinction gradients. The local-disk color gradients are small, however, and if present at high $\mathrm{z}$, would increase the observed sizes, rather than decrease them, as the $\mathrm{i}_{775}$ band shifts to the rest frame blue. The scale length also depends only slightly on bulge-disk decomposition, becoming smaller if the fitted exponential includes the bulge (Courteau 1996). This is not a consideration here because the median local slope of the profile avoids the steeper slope of the bulge region, as mentioned above, and because the UDF spirals do not have such prominent bulges as local early-type galaxies.

de Jong (1996a) and Beijersbergen, de Blok, \& van der Hulst (1999) noted that local galaxies have a slight correlation between extrapolated central disk surface brightness and exponential scale length in the sense that higher surface brightness galaxies are intrinsically smaller (see also McGaugh \& Bothun 1994). The difference in central surface brightness between local galaxies with a scale length of $3.5 \mathrm{kpc}$ (the average for the local-galaxy surveys quoted above), and local galaxies with a scale length of $1.5 \mathrm{kpc}$ (the average for our UDF spirals) corresponds to between 1 and $1.5 \mathrm{mag} \operatorname{arcsec}^{-2}$ in rest frame B band. Thus the small sizes observed here for UDF spirals might be the result of an observational bias toward high central surface brightness galaxies in the UDF. Independent evidence for a $\sim 25 \%$ loss of spirals larger than 10 pixels below the UDF sensitivity limit was presented elsewhere (Elmegreen, et al. 2005b), based partly on the distribution of axial ratios. The magnitude of the selection effect required for scale lengths seems too large to explain our UDF distribution, however. To get the small scale lengths, we would have to be selecting only UDF spirals that are intrinsically brighter than the face-on average at high redshift by $1-1.5$ mag $\operatorname{arcsec}^{-2}$ in the center. This contrasts with our previous study, where we estimated that the detection limit is only $0.25-0.5 \mathrm{mag} \operatorname{arcsec}^{-2}$ brighter than the average surface brightness. Larger galaxy losses at low surface brightness should shift the peak in the axial ratio distribution to values lower than $\sim 0.5$ (minor to major axis ratio), where it is now. This shift might be possible if internal dust removes such highly inclined galaxies from the UDF survey. The galaxies that are in our survey in fact have no correlation between central surface brightness and scale length (the two quantities make a scatter plot, which is not shown here). Thus the hypothesized large and low-surface brightness galaxies have to be missing already from our UDF survey. Nevertheless, it remains possible that UDF spirals appear small because of surface brightness or other selection effects. 
A small size for high redshift galaxies has been noted before (Lowenthal et al. 1997; Bouwens, Broadhurst, \& Silk 1998; Bouwens \& Silk 2002; Ferguson, Dickinson, \& Giavalisco 2004; Papovich, Dickinson, \& Giavalisco 2005; Böhm \& Ziegler 2005). Lyman Break galaxies at higher redshift are even smaller (Baugh et al. 1998; Somerville, Primack \& Faber 2001). Small exponential scale lengths $(\sim 2 \mathrm{kpc})$ were also found for $z \sim 0.8$ barred spirals in the Tadpole galaxy field (Elmegreen, Elmegreen \& Hirst 2004b). Disk scale lengths are expected to be smaller in galaxy evolution models in proportion to the dark matter virial radius, which varies as $H(z)^{-2 / 3} \sim(1+z)^{-1}$ for constant mass, Hubble parameter $H(z)$, and large z (Mo, Mau \& White 1998; Bouwens, Broadhurst, \& Illingworth 2003; Ferguson, Dickinson, \& Giavalisco 2004).

The scale length distribution function in Figure 3 is more revealing about galaxy size then the distribution of scale length versus luminosity. Trujillo et al. (2005) found relatively small scale lengths at high $\mathrm{z}$ for a given luminosity, but this may be the case regardless of absolute length because high redshift galaxies tend to have high surface brightnesses from high star formation rates; then a fixed luminosity covers a smaller radius. High intrinsic surface brightness is also expected from sampling effects even without higher star formation rates because surface brightness dimming hides the fainter populations.

The size evolution of a distribution of galaxies can differ from the size evolution of any particular galaxy. If there is little size evolution for particular galaxies, as suggested by Simard et al. (1999) and Ravindranath et al. (2004), then most of our spirals will become dwarfs or perhaps merge to form ellipticals. Only the largest members of our sample could turn into spirals today (e.g., Labbé et al. 2003).

One problem with this model of no size evolution is that the small star-forming galaxies observed locally, which are typically types Im, Sm, or BCD, do not have such clear spirals as the small UDF spiral galaxies. The local dwarf disks are relatively thick (van den Bergh 1988) and their stellar velocity dispersions relatively large compared to their small rotation speeds, so they have little ability to sustain spiral waves. Thus, distant spirals are distinct from local dwarfs even at the same physical size.

To sustain a global spiral wave, the Toomre (1964) length, $2 \pi G \Sigma / \kappa^{2}$ (for epicyclic frequency $\kappa$ and total disk surface density $\Sigma$ ) has to be comparable to the disk scale length. The Toomre length is essentially the separation between stellar spiral arms. To generate a strong wave while avoiding catastrophic collapse, the Toomre (1964) instability parameter, $Q=\kappa c /(3.36 G \Sigma)$ for stellar velocity dispersion $c$ has to be between 1 and 3 . If the disk scale length is small for a galaxy that is morphologically spiral but has a high surface brightness, then $\kappa$ has to be large. The stability parameter then requires $c$ to be fairly small, which is possible if the disk mass is dominated by gas. For the UDF spirals, these constraints would 
be satisfied if the visible disks are the inner gassy regions of fairly dense halo potentials, where the density is about the same as in the inner regions of spiral galaxies today. For a flat or solid body inner rotation curve, $\kappa$ scales with the square root of the total enclosed density. At high redshift, galaxies should be not only smaller but also denser in some inverse proportion (Mo, Mau \& White 1998), allowing tiny spirals to appear somewhat normal.

The Toomre length may also be viewed in another way. The ratio of the Toomre length to the galaxy size is comparable to the ratio of the disk mass to the total mass in the disk region. Thus, the appearance of global spirals implies that disk masses are comparable to halo masses out to the same radius. This is known to be true for local spiral galaxies. Our observation of high redshift spirals implies it is true there too, regardless of their size. The situation is different for local dwarf irregulars, however. There the ratio of disk mass to halo mass is relatively small, i.e., they have relatively massive dark halos (e.g., Carignan \& Beaulieu 1989; Persic \& Salucci 1995). It follows that the Toomre length is small compared to the galaxy size in dwarfs, and so a stellar disturbance makes relatively small epicycles and no global density waves.

These considerations lead us to believe that small UDF spirals are dense like the inner regions of modern spirals. This should be the case even if we select only the small members of a larger sample because of surface brightness bias or other effects (see above). The small spirals should grow to become large spirals by adding low-density halo material to the periphery over time and by filling out their disks with accreted gas and radially increasing star formation (if they are not cannibalized first). This is the commonly discussed inside-out formation model of galaxy disks but now with an increasing scale length in addition to an increasing overall size. A similar conclusion was reached by Papovich et al. (2005) on the basis of an observed increase in half-light radius and color gradient for $z \sim 1$ compared to $z \sim 2.3$ galaxies in the Hubble Deep Field North. The color gradient in the $z \sim 1$ galaxies was in the sense of a red core and a blue envelope, as if star formation moved outward. Spiral arms were not so readily resolved in that survey compared to the UDF, but some of the galaxies studied by Papovich et al. are clearly spirals. They also point out that for such color gradients to exist, the major merger activity has to be mostly finished by $z \sim 1$, a conclusion also reached by others for different reasons (e.g., Conselice, Blackburne \& Papovich 2005).

The higher density of high-redshift spirals has implications for the formation and evolution of central bars. Bar formation is faster at higher density, and in a gas-rich young galaxy, also more dissipative than usually considered in N-body simulations of modern stellar disks. Bar destruction with a central mass concentration and gas torques should be faster at early times too, for the same reasons. Moreover, if a fully-grown nuclear black hole is present at 
this stage, then the disk mass fraction represented by the black hole will be larger than it is for a modern disk, thus promoting bar destruction by the degeneration of $\mathrm{x}_{1}$ orbits (e.g., Hasan, Pfenniger, \& Norman 1993). Modern disks do not have the central mass concentration necessary for such bar destruction (Shen \& Sellwood 2004; Athanassoula, Lambert, \& Dehnen 2005), but very young disks could have. For these reasons, it might be possible for young spiral disks that are small, dense, and gas-rich to form and disperse relatively small bars in their centers, thereby quickening the build up a bulge from the disk.

\section{Clump Luminosity Fractions in UDF Disk Galaxies}

Galaxies at high redshift typically have giant blue clumps that are associated with recent star formation. In spirals, these clumps are often in the arms, as they are in modern galaxies, while in clump clusters, they are dispersed throughout the disk. Equally large clumps are in chain galaxies (Cowie, Hu, \& Songaila 1995), high redshift-elliptical galaxies (Elmegreen, Elmegreen, \& Ferguson 2005), tadpole galaxies (van den Bergh et al. 1996), and doubleclump galaxies (van den Bergh 2002). A review of the various morphologies seen in the UDF is in Elmegreen et al. (2005b).

Clump clusters differ from spiral galaxies not only in the regularity of the clump positions, but also in the fraction of the total light that is in the form of clumps. Figure 5 shows histograms of this fraction using magnitudes at $i_{775}$. Magnitudes were determined in IRAF using a central aperture of 3 pixels radius and a background subtraction annulus of 5 pixels width with a separation of 2 pixels between them. The lower two panels are for the 91 relatively face-on spiral galaxies in the UDF, with the bottom panel including the central clumps or bulges in these galaxies and the middle panel excluding the bulges. The bulges are generally more luminous than the other clumps in the spirals, so the clump fraction is much higher when the bulges are included. The top panel is for all 178 clump clusters, which have a very high fraction of their total flux in the form of clumps. The average flux fractions are $0.27 \pm 0.14$ for clump clusters, $0.16 \pm 0.10$ for spirals including their bulges, and $0.080 \pm 0.065$ for spirals not including their bulges. Ten extreme cases of clump clusters were studied in Elmegreen \& Elmegreen (2005) in which an average of $40 \%$ of the $i_{775}$ flux was in the clumps.

The $\mathrm{i}_{775}$ band corresponds to a different rest wavelength for each galaxy, and the clump flux fraction is generally higher at shorter wavelengths for local galaxies (i.e., star-forming regions are blue). Thus one could interpret Figure 5 as an indication that the clump clusters

have systematically higher redshifts than the spirals, with the shorter rest wavelengths more clumpy. However, the large clump fraction at short wavelengths for local galaxies is the result of a big difference between clump and disk colors, the clumps being much bluer. This 
difference is not as large at high redshift because the interclump stars are not as old as they are locally. The average $B_{435}-V_{606}$ and $V_{606}-i_{775}$ color differences between clump and interclump emissions in the 10 clump cluster galaxies studied earlier are $0.19 \pm 0.17 \mathrm{mag}$ and $0.14 \pm 0.30 \mathrm{mag}$, respectively, with the clumps being bluer than the interclump regions. Similarly, the $\mathrm{V}_{606}-\mathrm{I}_{814}$ difference between the clump and surrounding emission for the chain and spiral galaxies in the Tadpole field is $0.2-0.3$ mag (Elmegreen, Elmegreen, \& Sheets 2004). In contrast, the difference between clump and average colors in local spiral galaxies is much greater. Local spiral galaxies have total $B-V \sim 0.7 \pm 0.1$ depending on Hubble type, and star forming regions with $B-V \sim 0$, so the clumps are bluer than the average by $\sim 0.7 \mathrm{mag}$. The clump-interclump color difference is even larger for local galaxies because the interclump emission is redder than the average.

We consider in this paper that the difference in clump flux fraction between spiral and clump cluster galaxies is a reasonable indication that there is a real difference in the intrinsic clumpiness of the two types of galaxies. A more quantitative measure of disk clumpiness will require redshift information.

\section{Clump Position Distribution in UDF Disk Galaxies}

To study the relation between clump positions and exponential disks, the (x,y) positions and $\mathrm{B}, \mathrm{V}, \mathrm{i}$, and $\mathrm{z}$ magnitudes of all the bright clumps in the low-inclination spiral and clump cluster galaxies of the UDF were determined individually using IRAF. This represents 632 objects (including bulges) in the spirals and 904 objects in the clump clusters. The centers of all the galaxies were measured also, using the middle of the central peak for the spirals and the geometrically centered position of the outer $2 \sigma$ contour for the clump clusters. Deprojected galactocentric radii were then determined from these positional measurements, assuming a circular disk-like geometry with an inclination from the axial ratio.

Because the galaxies span a wide range of redshifts, we normalized the radii to the exponential scale length in the spirals, and to the outer $2 \sigma$ isophotal radius for both the spirals and the clump clusters. This normalization also makes the rest wavelength of the measurement relatively unimportant (it differs for each galaxy) because the radial distribution of intensity is effectively determined only for each galaxy separately and then added together for all galaxies. Different rest wavelengths do not affect the relative distribution of intensity inside each disk as long as there are no systematic correlations between clump age and radius. In fact, clump B-V color has no measurable correlation with deprojected radius for either galaxy type (not shown here). 
Figure 6 plots the $\mathrm{i}_{775}$ magnitudes of the clumps versus the normalized galactocentric radii. The points at zero radius for the spirals are the bulges. The clumps get fainter further from the center. The dotted line in the lower panel has unit slope, corresponding to the magnitude-radius relation of each underlying exponential disk. The distribution of points lies parallel to this dotted line, suggesting a selection effect: we can measure only the clumps that stand out sufficiently above the background disk. Such an effect seems likely because star-forming clumps in local galaxies have a wide range of luminosities with a power-law distribution function dominated in number by the smallest members. Thus, there may be countless small clumps that were not measured. However, this should not be a problem for our analysis because the local power laws have slopes of $\sim-2$ or shallower for linear intervals of luminosity, and this means that the integrated luminosity in all of the clumps in a particular radial range is some fixed factor (less than $\sim 5$ ) times the luminosity of the largest member. The fact that there are no high luminosity clumps in the outer regions (the lower envelopes in Fig. 5 also increase with radius) means that there really is an overall gradient in clump properties, although we may not be seeing the true gradient with our selection effect. This conclusion is supported by the observation that there is also a tendency for the clumps to get fainter with galactocentric radius in clump cluster galaxies. There are no significant underlying disks in these cases and no likely systematic confusion about clump definition and brightness at any radius.

Figure 7 shows the radial distributions of clump number density and clump flux density, converted to a magnitude scale. The lower panels are for the clump number densities. These were determined by summing the number of clumps in all galaxies of each type that lie between radial intervals separated by 0.1 in normalized units. On the left, the normalization of radius is with the exponential scale length (for the spirals only) and on the right, the normalization is with the radius of the outer $2 \sigma$ contour. The top panels are for the clump flux densities. These were determined by summing the $i_{775}$ fluxes, $10^{-0.4 m_{I}}$, for all the clumps that lie between the same normalized radial intervals. In both cases, the sums were divided by the areas of the annuli corresponding to the radial interval and then converted to a (negative) magnitude scale by the operation $2.5 \log$ (sum/area). The dotted lines on the left have unit slope, corresponding to the exponential disks in each spiral galaxy. The vertical scale in the figure has an arbitrary zero point with higher densities toward the top.

The clump distributions in the spiral and clump cluster galaxies show a remarkable similarity to each other even though the radial profiles of the two types differ significantly in individual cases. This similarity is shown best by the agreement between the dashed and solid lines in the right-hand panels. Evidently, the clumps in clump cluster galaxies are mapping out an exponential disk that is just like the exponential in spiral galaxies. This implies that if the clumps in clump cluster galaxies are blended together, by dispersal of 
the associated giant star complexes, for example, then an exponential disk will result that is essentially the same as the disk in a spiral galaxy. This similarity does not extend to the bulges, however: the average density of clump fluxes at the centers of spirals is higher than it is at the centers of clump clusters by 2 magnitudes (a factor of 6 in the top right panel). The bulges can be seen again as the sharp rise at zero radius in the left-hand panels.

The correlation between clump flux and normalized galactocentric radius (Fig. 6) implies that the radial distribution of average clump flux density (top of Fig. 7) is slightly steeper than the radial distribution of clump number density (bottom of Fig. 7). For radii between $1.5 R_{\text {disk }}$ and $5 R_{\text {disk }}$, the slope of the distribution on the left-hand plot of number density is -1.3 and the slope of the distribution on the left-hand plot of flux density is -1.8 . Thus the integrated flux from clumps in the spiral galaxies has an exponential scale length equal to $1 / 1.8=0.55$ times the scale length of the total light. Dispersing the clumps will shorten the overall scale length slightly (in proportional to the relative luminosity of the clumps compared to the total disk).

\section{Conclusions}

The exponential scale lengths for spiral galaxies in the UDF appear to be a factor of $\sim 2$ smaller than for local spiral galaxies. Selection effects could give this result but the required high central surface brightness seems more extreme than permitted by the distribution of the ratio of axes. Because the presence of global spiral waves implies that stellar disks are relatively massive compared to the total mass inside the disk radius, the small spiral galaxies in the UDF are probably only the dense inner regions of today's disks. Subsequent accretion at lower density should build up both the disk and the halo over time, increasing the scale length and the overall galaxy size. Bars that form early in dense, gas-rich disks would be more prone to orbit degeneration and dispersal into a bulge than bars which form later in fully developed disks. N-body simulations of bar formation should be revised to include more gas and to use smaller, denser initial disks.

Galaxies in the UDF, including the spirals, are highly clumped into blue star-forming regions that contain up to $\sim 10^{9} \mathrm{M}_{\odot}$ of stars $\sim 1$ Gy old or younger. The most clumpy of the disk galaxies are called clump-clusters. In the low-inclination clump-cluster galaxies studied here, $0.27 \pm 0.14$ of the total $i_{775}$ flux comes from giant disk clumps, compared to only $0.080 \pm 0.065$ for the spirals. In a previous paper, we suggested that many of these

clumps should be dispersing as a result of tidal forces. Here we addressed the question of what may happen to the clumps after they disperse. 
The evidence suggests that the clumps in clump-cluster galaxies disperse into a more uniform disk that has an exponential radial light profile. We found that the clumps in both spirals and clump-clusters were distributed in an exponential fashion, and that their total

flux was also distributed as an exponential. Even though clump cluster galaxies do not presently have an exponential light profile in a smooth disk, their average clump distribution is as exponential as the profile in a spiral galaxy.

A similar conclusion was based on the near-exponential nature of the average radial profiles of clump cluster galaxies made from elliptical contours. While the major axis or single-cut profiles are highly irregular, the average radial profiles, averaged over projected ellipses in azimuth, are closer to exponential in many cases (Fig. 1).

We also showed that spiral galaxy bulges stand out above the exponential profile and are not part of it. Clump clusters generally do not have such bulges. Thus it remains to be determined whether clump clusters form primarily late Hubble-type disks over time, or whether some of the clumps merge into a bulge, as in models by Immeli et al. (2004).

Acknowledgments: We are grateful to the referee for comments that improved the presentation of this paper. B.G.E. is supported by the National Science Foundation through grant AST-0205097.

\section{REFERENCES}

Abadi, M.G., Navarro, J.F., Steinmetz, M., Eke, V.R. 2003, ApJ, 597, 21

Athanassoula, E., Lambert, J.C., \& Dehnen, W. 2005, MNRAS, in press

Baugh, C.M., Cole, S., Frenk, C.S., \& Lacey, C.G. 1998, ApJ, 498, 504

Beckwith, S., et al. 2005, in preparation

Beijersbergen, M., de Blok, W. J. G., \& van der Hulst, J. M. 1999, A\&A, 351, 903

Böhm, A., \& Ziegler, B.L. 2005, Rev. Mod. Astron., 18, 106

Bouwens, R., Broadhurst, T., \& Silk, J. 1998, ApJ, 506, 557

Bouwens, R., Broadhurst, T., \& Illingworth, G. 2003, ApJ, 593, 640

Bouwens, R., \& Silk, J. 2002, ApJ, 568, 522

Brook, C.B., Kawata, D., Gibson, B.K., \& Freeman, K.C. 2004, ApJ, 612, 894 
Carignan, C., \& Beaulieu, S. 1989, ApJ, 347, 760

Conselice, C., Grogin, N.A., Jogee, S., Lucas, R.A., Dahlen, T., de Mello, D., Gardner, J.P., Mobasher, B., Ravindranath, S. 2004, ApJ, 600, L139

Conselice, C. J., Blackburne, J. A., Papovich, C. 2005, ApJ, 620, 564

Courteau, S. 1996, ApJS, 103, 363

Cowie, L., Hu, E., \& Songaila, A. 1995, AJ, 110, 1576

de Blok, W., van der Hulst, J., \& Bothun, G. 1995, MNRAS, 274, 235

de Grijs, R. 1998, MNRAS, 299, 595

de Jong, R.S. 1996a, A\&A, 313, 45

de Jong, R.S. 1996b, A\&A, 313, 377

Elmegreen, D.M., Elmegreen, B.G., \& Sheets, C. 2004, ApJ, 603, 74

Elmegreen, D.M., Elmegreen, B.G., \& Hirst, A.C. 2004a, ApJ, 604, L21 (Paper II)

Elmegreen, B.G., Elmegreen, D.M., \& Hirst, A.C. 2004b, ApJ, 612, 191 (Paper III)

Elmegreen, B.G., \& Elmegreen, D.M. 2005, ApJ, 627, 632

Elmegreen, D.M., Elmegreen, B.G., \& Ferguson, T.E. 2005, ApJ, 623, L71

Elmegreen, D.M., Elmegreen, B.G., Rubin, D.S., \& Schaffer, M.A. 2005b, ApJ, 631, 85

Erwin, P., Beckman, J. E., \& Pohlen, M. 2005, ApJL, 626, 81

Ferguson, A.M.N., \& Clarke, C.J. 2001, MNRAS, 325, 781

Ferguson, H.C., Dickinson, M., Giavalisco, M., 2004, ApJ, 600, L107

Freeman, K.C. 1970, ApJ, 160, 811

Governato, F., Mayer, L., Wadsley, J., Gardner, J. P., Willman, B., Hayashi, E., Quinn, T., Stadel, J., \& Lake, G. 2004, ApJ, 607, 688

Hasan, H., Pfenniger, D. \& Norman, C. 1993, ApJ, 409, 91

Hunter, D.A., \& Elmegreen, B.G. 2005, ApJS, 162, preprint doi:10.1086/498096

Immeli, A., Samland, M., Westera, P., \& Gerhard, O. 2004, ApJ, 611, 20 
Keres, D., Katz, N., Weinberg, D.H., \& Davé, R. 2005, MNRAS, in press, astroph/0407095

Kregel, M., \& van der Kruit, P. C. 2004, MNRAS, 355, 142

Labbé, I., et al. 2003, ApJ, 591, L95

Lin, D.N.C., \& Pringle, J.E. 1987, ApJL, 320, 87

Lowenthal, J.D., Koo, D.C., Guzmán, R., Gallego, J., Phillips, A.C., Faber, S.M., Vogt, N.P., Illingworth, G.D., \& Gronwall, C. 1997, ApJ, 481, 673

McGaugh, S.S., \& Bothun, G.D. 1994, AJ, 107, 530

Mo, H.J., Mao, S., \& White, S.D.M. 1998, MNRAS, 295, 319

Murali, C., Katz, N., Hernquist, L., Weinberg, D.H., \& Davé, R. 2002, ApJ, 571, 1

O’Neil, J., Bothun, G.D., \& Impey, C.D. 2000, ApJS, 128, 99

Papovich, C., Dickinson, M., Giavalisco, M. 2005, ApJ, 631, 101

Pérez, I. 2004, A\&A, 427, L17

Persic, M. \& Salucci, P. 1995, ApJS, 99, 501

Ravindranath, S., et al. 2004, ApJ, 604, L9

Robertson, B., Yoshida, N., Springel, V., Hernquist, L. 2004, ApJ, 606, 32

Robertson, B., Hernquist, L., Bullock, J.S., Cox, T.J., Di Matteo, T., Springel, V., Yoshida, N. 2005, astroph/0503369

Shen, J., \& Sellwood, J.A. 2004, ApJ, 604, 614

Simard, L., Koo, D.C., Faber, S. M., Sarajedini, V. L., Vogt, N. P., Phillips, A. C., Gebhardt, K., Illingworth, G. D., \& Wu, K. L. 1999, ApJ, 519, 563

Somerville, R.S., Primack, J.R., Faber, S.M. 2001, MNRAS, 320, 504

Sommer-Larsen, J., Götz, M., \& Portinari, L. 2003, ApJ, 596, 47

Spergel, D.N., et al. 2003, ApJS, 148, 175

Swaters, R. \& Balcells, M. 2002, A\&A, 390, 863 
Toft, S., van Dokkum, P., Franx, M., Thompson, R. I., Illingworth, G. D., Bouwens, R. J., \& Kriek, M. 2005, ApJ, 624, L9

Toomre, A. 1964, ApJ, 139, 1217

Trujillo, I., Forster Schreiber, N.M., Rudnick, G., et al. 2005, astroph/0504225

van den Bergh, S. 1988, PASP, 100, 344

van den Bergh, S. 2002, PASP, 114, 797

van den Bergh, S., Abraham, R.G., Ellis, R.S., Tanvir, N.R.,Santiago, B.X., \& Glazebrook, K.G. 1996, AJ 112, 359

van den Bosch, F.C. 2001, MNRAS, 327, 1334

van der Kruit, P.C. 1987, A\&A, 173, 59

Walker, I., Mihos, J., \& Hernquist, L. 1996, ApJ, 460, 121

Westera, P., Samland, M., Buser, R., \& Gerhard, O.E. 2002, A\&A, 389, 761

Yoshii, Y. \& Sommer-Larsen, J. 1989, MNRAS, 236, 779

Zhang, B., Wyse, R.F.G. 2000, MNRAS, 313, 310 


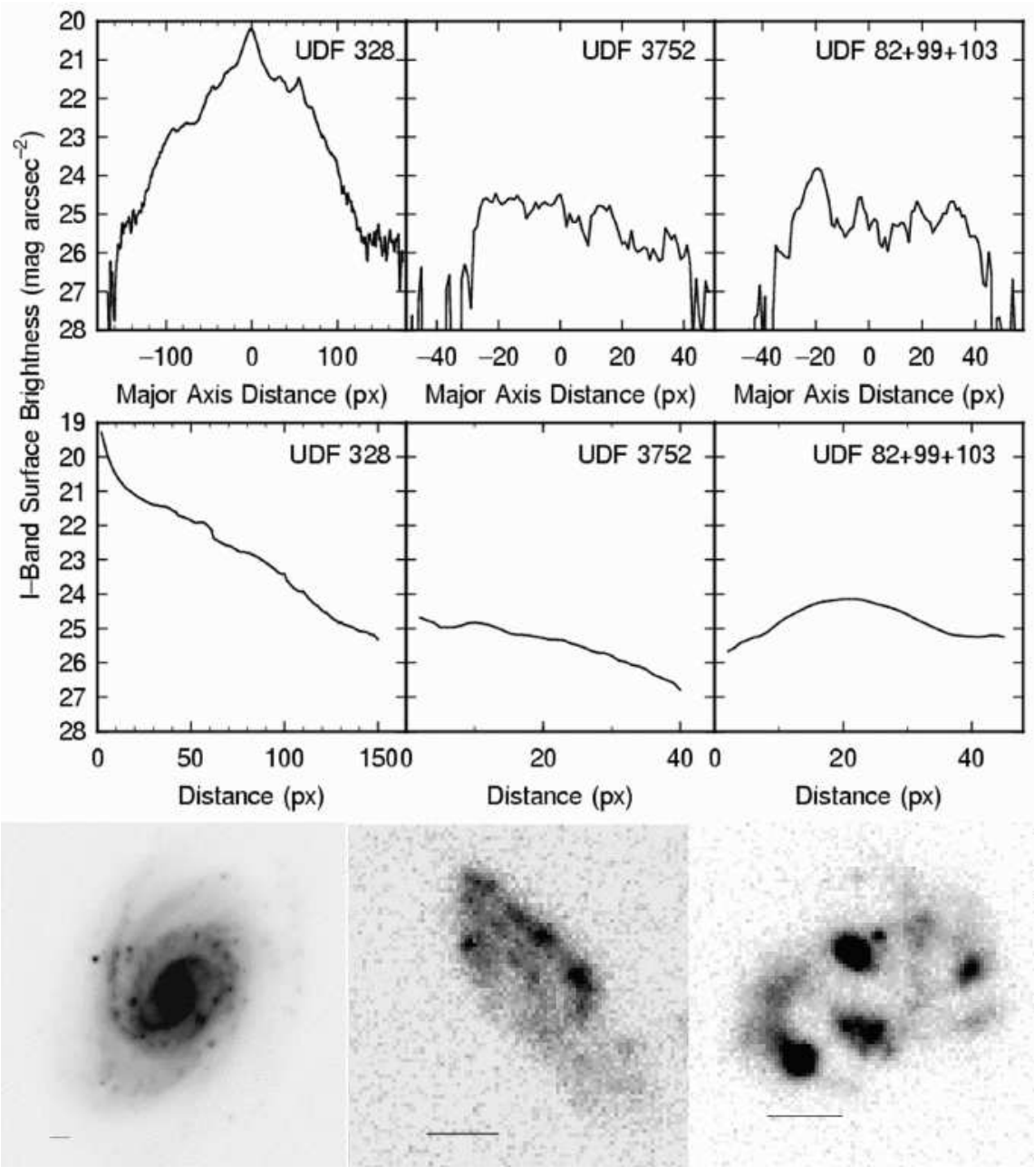

Fig. 1.- A spiral galaxy (UDF 328) and two clump cluster galaxies (UDF 3752 in the middle column and UDF $82+99+103$ on the right) are shown, with major axis profiles on the top and elliptical-average radial profiles in the middle. UDF 328 has an exponential disk in both profiles, which is typical for spirals, but the two clump-clusters, like most in their class, have no exponential profiles along the major axes. The average profiles for clump cluster galaxies may or may not be exponential. UDF 3752 has an average profile that is exponential and UDF $82+99+103$ has one that is not. The line in each grayscale image represents 0.5 arcsec. 

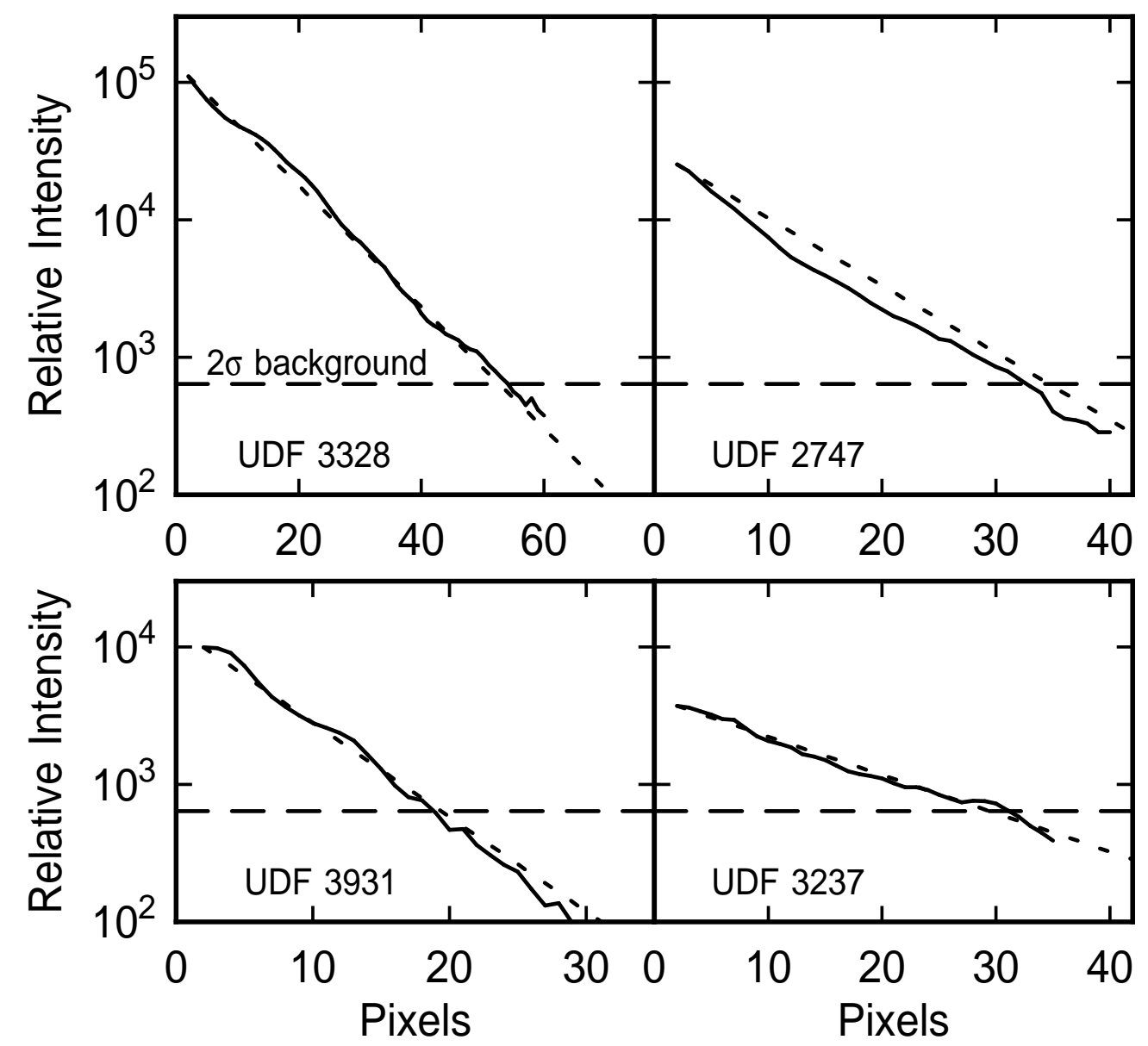

Fig. 2.- Four sample exponential disk fits using the median technique. 


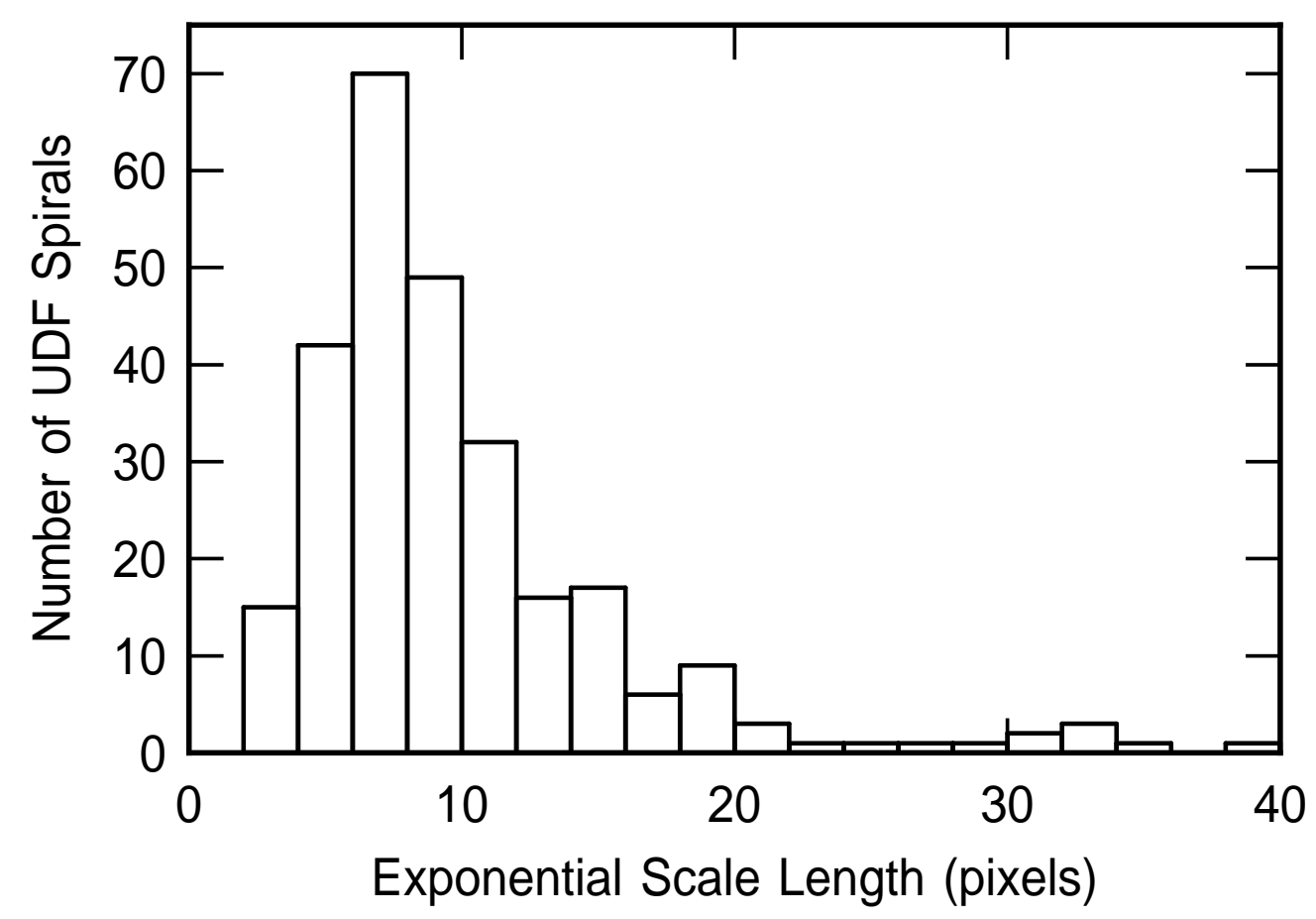

Fig. 3.- The distribution of exponential scale lengths in spiral galaxies of the UDF. The length is plotted in units of a UDF pixel, which is 0.03 arcsec. 


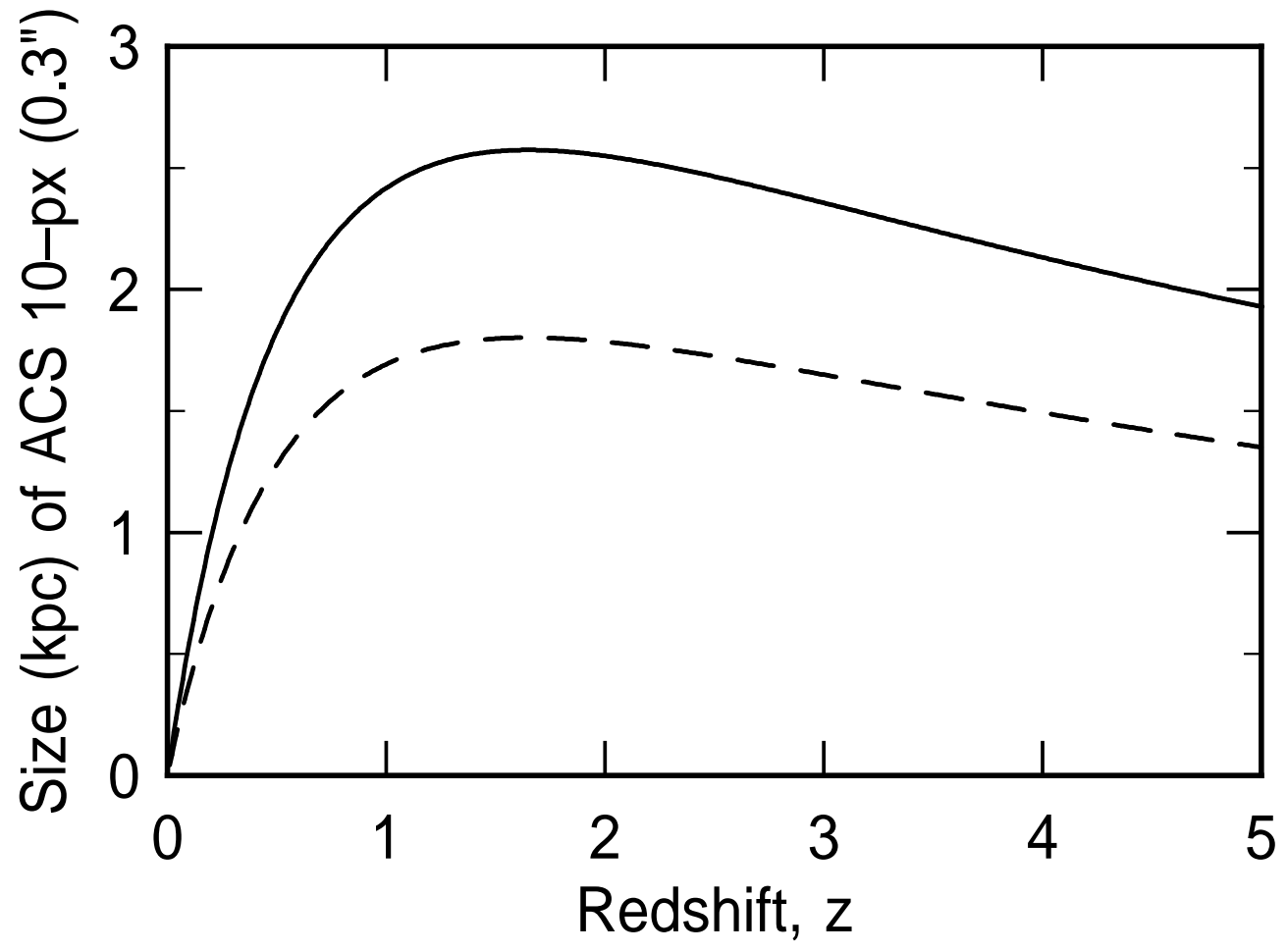

Fig. 4.- The physical size of a region that subtends 10 pixels (solid line) or 7 pixels (dashed line) of the ACS UDF field is plotted as a function of cosmological redshift for the WMAP cosmology. The conversion between angle and physical size is relatively insensitive to $z$ in the range from 0.5 to 5 , allowing us to interpret the distribution of angular scale lengths shown in the previous figure into an approximate distribution of physical scale lengths. 


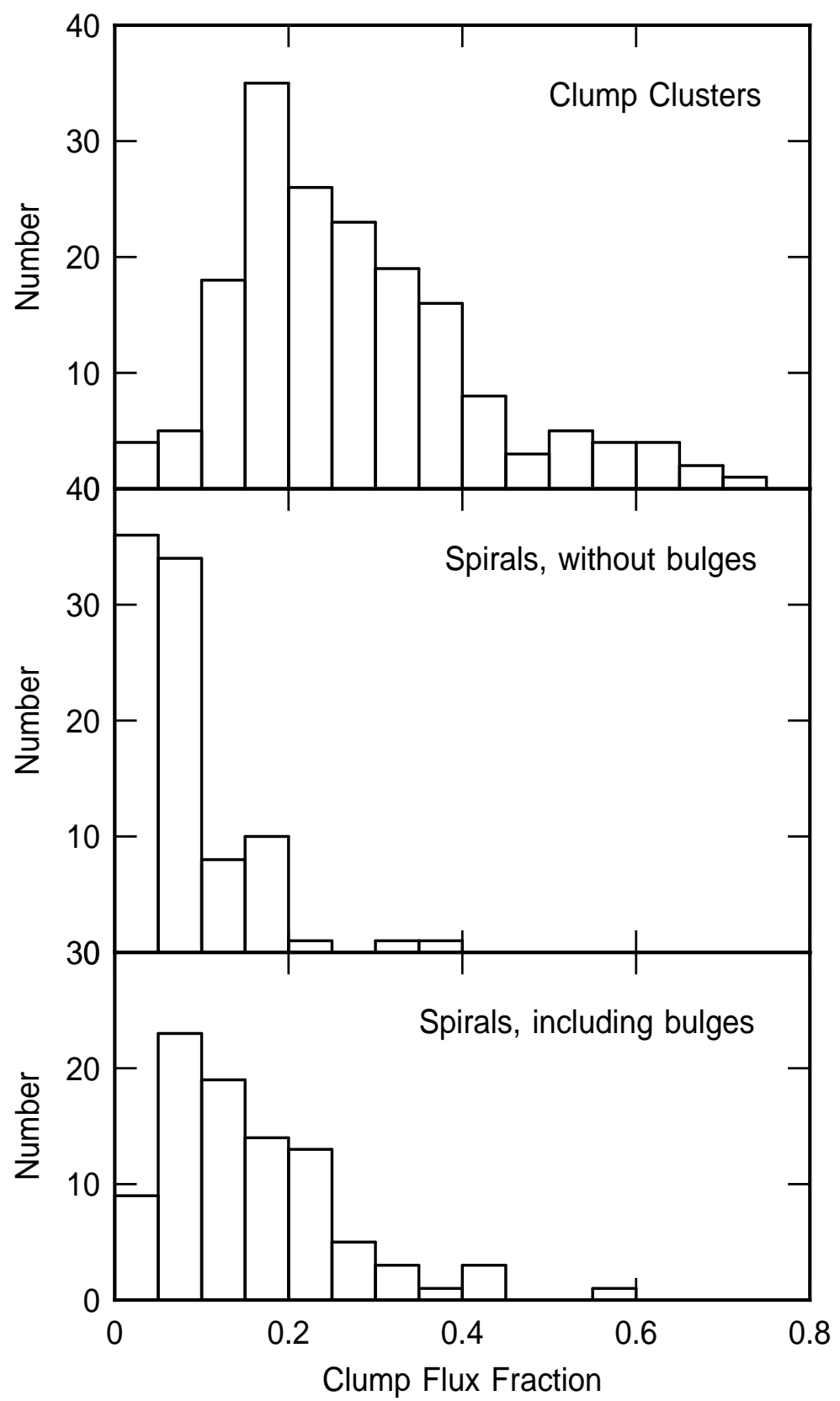

Fig. 5.- Histograms of the fraction of the total galaxy light in the form of giant clumps, determined at $i_{775}$. The lower panel is for spirals where the clumps include the central bulge; the middle panel is for spirals without the bulges. The top panel is for clump cluster galaxies. The clump clusters have $0.27 \pm 0.14$ of their measured $\mathrm{i}_{775}$ flux in the form of giant clumps while the spirals have only $0.080 \pm 0.065$ of their emission in such clumps, excluding the bulges. 

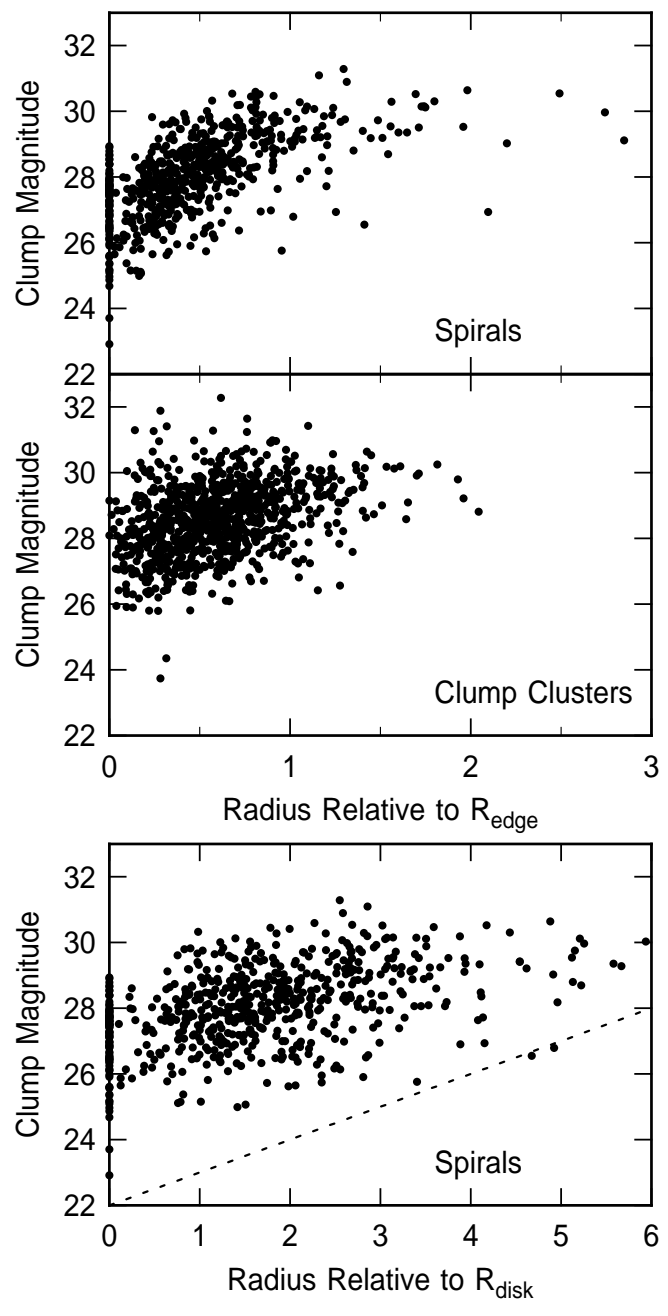

Fig. 6.- The distribution of clump magnitudes in spiral and clump-cluster galaxies based on an IRAF aperture 3 pixels in radius. The clumps get fainter with deprojected distance from the galaxy center, nearly in proportion to the background exponential disk for the case of spirals (lower panel, dotted line), but also for the clump clusters where there is relatively little background disk light. The top two panels have galacto-centric radius normalized to the semi-major axis of the outer isophotal contour, which is at a level of $2 \sigma$ for noise $\sigma$. The bottom panel has the radius normalized to the spiral scale length. 

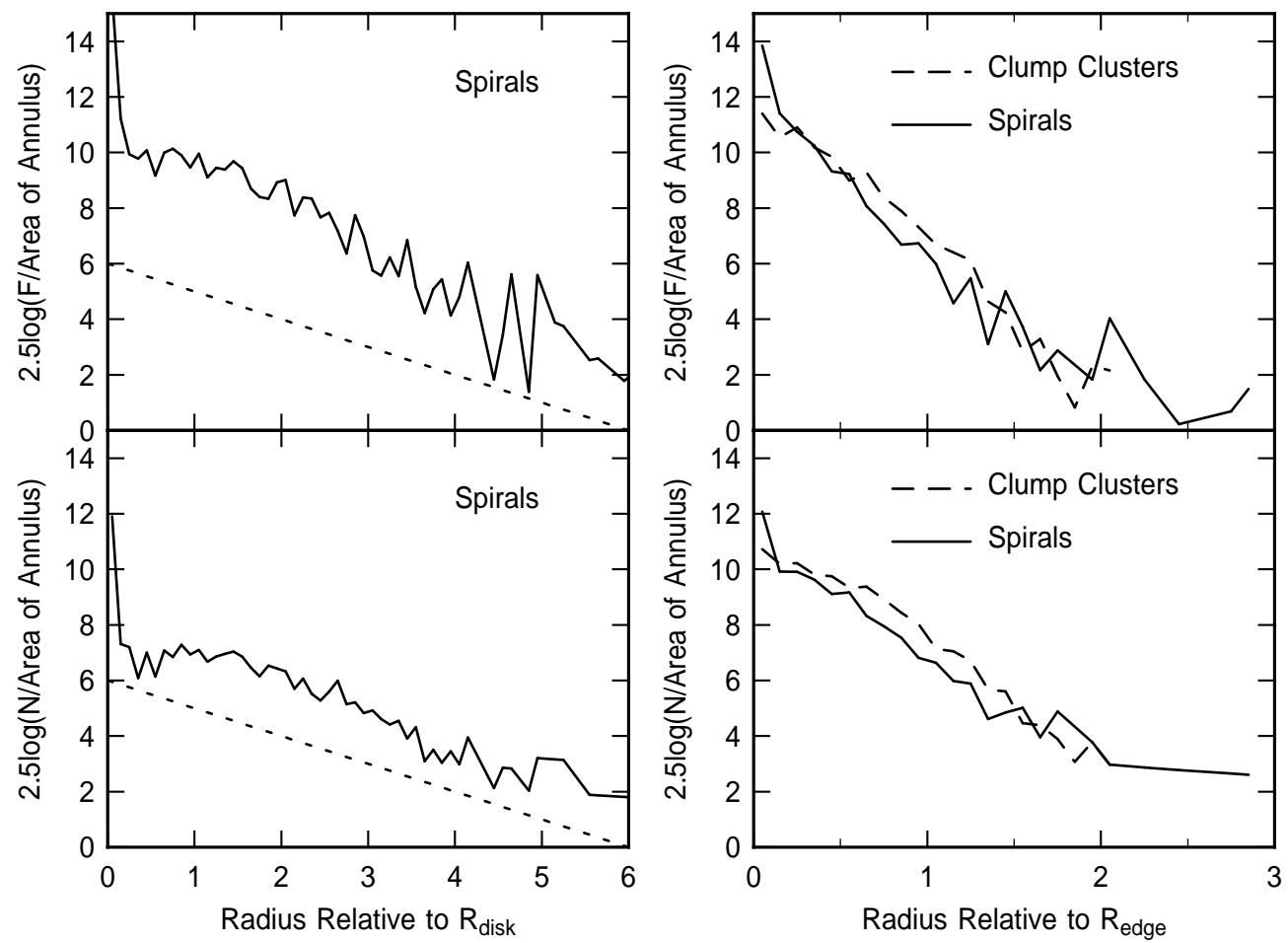

Fig. 7.- The distribution with normalized galactocentric radius of the clump number density (bottom) and the total clump flux density (top) in spiral and clump-cluster galaxies. The normalization is with the disk scale length on the left (only for spirals) and with the semimajor axis of the outer $2 \sigma$ isophotal contour on the right. The distribution of clumps follows the background exponential disk in the case of spirals. The distributions for clump clusters and spirals are about the same. This result implies that dispersing clumps will convert the highly irregular disk of a clump-cluster galaxy into one that is more like a spiral galaxy with an exponential disk. 